\title{
Análise da rejeição nos pacientes transplantados por anemia aplástica severa condicionados com ciclofosfamida ou a associação desta ao bussulfano
}

\author{
Analysis of rejection in transplanted patients suffering severe aplastic anemia conditioned with \\ cyclophosphamide alone or associated with busulfan
}

Lidice C. Lenz e Silval

Ricardo Pasquini ${ }^{2}$

\begin{abstract}
O transplante de medula óssea é um tratamento eficaz para pacientes com anemia aplástica severa (AAS) e é a modalidade terapêutica de escolha para pacientes jovens com doador aparentado HLA idêntico. A rejeição é uma importante complicação do transplante de medula, que, independentemente do tipo de tratamento imunossupressor pré e pós-transplante, ocorre em $55 \%$ a $60 \%$ dos pacientes. O serviço de TMO da Universidade Federal do Paraná (UFPR) acumula a experiência de 178 casos de AAS transplantados no periodo de 1993 a 2001, usando como condicionamento tanto a ciclofosfamida (CFA) como a combinação desta ao bussulfano $(C F A+B U)$. Dentre eles, 39 apresentaram rejeição ou falha de pega. Dos pacientes condicionados com ciclofosfamida, 24 (46\%) apresentaram rejeição, sendo 3 (6\%) com falha primária de pega (FPP) e 21 (40\%) com pega transitória (PT). Entre os pacientes condicionados com BU+CFA, 15 (12\%) apresentaram rejeição, sendo 4 (3\%) com FPP e 11 (9\%) com pega transitória. Os pacientes condicionados com ciclofosfamida $(200 \mathrm{mg} / \mathrm{kg})$ que apresentaram rejeição tiveram uma sobrevida global alta (aproximadamente 80\%), pois conseguiram ser resgatados por um novo transplante ou pelo tratamento imunossupressor com ciclosporina. A sobrevida dos pacientes politransfundidos condicionados com a associação de ciclofosfamida e bussulfano foi de aproximadamente 35\%. Rev. bras. hematol. hemoter. 2005;27(1):5-11.
\end{abstract}

Palavras-chave: Transplante de medula óssea; anemia aplástica severa; rejeição; falha primária de pega; ciclofosfamida; bussulfano.

\section{Introdução}

O transplante de medula óssea é um tratamento eficaz para pacientes com anemia aplástica severa (AAS) e é a modalidade terapêutica de escolha para pacientes jovens que tenham um doador aparentado HLA idêntico..$^{1-3}$ Os artigos analisados para a confecção do presente trabalho mostram que há sobrevida de $55 \%$ a $80 \%$ dos casos. ${ }^{4-8}$

A rejeição é uma importante complicação do transplante de medula, que, independentemente do tipo de tratamento imunossupressor pré e pós-transplante, ocorre em 55\% a $60 \%$ dos pacientes submetidos ao TMO HLA idêntico. ${ }^{6,9-10}$

Este é um trabalho retrospectivo e tem como objetivo analisar o índice de rejeição dos pacientes transplantados por anemia aplástica severa no Hospital de Clínicas da Universidade Federal do Paraná (UFPR), bem como identificar os fatores que podem estar relacionados a este índice.

\section{Casuística e Método}

De janeiro de 1993 a novembro de 2001 foram realizados no Hospital de Clínicas da Universidade Federal do

\footnotetext{
${ }^{I}$ Médica hematologista, mestranda da UFPR.

Correspondência para: Lidice C. Lenz e Silva

Rua Dr. Romuldo 189/601 - São Mateus

36016-380 - Juiz de Fora-MG - Brasil

Tel: (32) 9987-7701

E-mail: lidicelenz@globo.com
}

${ }^{2}$ Médico, Professor Livre-Docente e Chefe do Serviço de Transplante de Medula Óssea da Universidade Federal do Paraná (UFPR). 
Paraná (UFPR) 205 transplantes alogênicos aparentados e totalmente compatíveis em pacientes portadores de AAS usando-se diferentes tipos de condicionamento. Destes, foram analisados 178 pacientes que apresentaram sobrevida superior a 21 dias, sendo que 52 deles foram condicionados com ciclofosfamida (CFA) e 126 com a combinação de bussulfano e ciclofosfamida (BU+CFA).

Os critérios para definição da AAS foram estabelecidos por Camitta e colaboradores em 1975. ${ }^{11}$

A partir de 1993 seguiu-se o protocolo no qual os pacientes com menos de 15 transfusões eram condicionados com dose total de $200 \mathrm{mg} / \mathrm{kg}$ de ciclofosfamida $(50 \mathrm{mg} / \mathrm{kg} / \mathrm{dia}$, durante quatro dias, do dia -6 ao -3 ) e os pacientes com 15 ou mais transfusões eram condicionados com $12 \mathrm{mg} / \mathrm{kg}$ de bussulfano (dividido em 16 doses e administrado nos dias -8 , $-7,-6$ e -5 ) associado a $120 \mathrm{mg} / \mathrm{kg}$ de ciclofosfamida (dividida em duas doses e administrada nos dias -4 e -3). Como exceção, cinco pacientes receberam mais de 15 transfusões, mas, por apresentarem estado geral comprometido, optou-se pelo condicionamento com ciclofosfamida, em razão da sua menor toxicidade. $\mathrm{O}$ número de unidades transfundidas nestes pacientes foi de 16, 18, 22, 28 e 30. A medula óssea foi infundida no dia zero.

A imunoprofilaxia da doença do enxerto contra o hospedeiro (DECH) foi feita com methotrexate (MTX) na dose de $15 \mathrm{mg} / \mathrm{m}^{2}$ no $\mathrm{D}+1$ e $10 \mathrm{mg} / \mathrm{m}^{2}$ nos dias $+3,+6 \mathrm{e}+11$ associado a ciclosporina (CSA) na dose de $1,5 \mathrm{mg} / \mathrm{kg}$ a cada 12 horas, por via endovenosa e iniciada no D-1. Quando o paciente conseguia ingerir a CSA por via oral, a dose era modificada para $6,25 \mathrm{mg} / \mathrm{kg}$ a cada 12 horas e mantida por um ano. As doses de CSA foram alteradas dependendo do nível sérico da droga e da concentração da creatinina sangüínea.

Como profilaxia da infecção pulmonar por Pneumocystis carinii usava-se o sulfametoxazol-trimetropim do dia -8 até o -1 e, a seguir, do D+50 ao D+150, de 12 em 12 horas, duas vezes na semana. A profilaxia contra infecção fúngica foi feita com anfotericina B $0,1 \mathrm{mg} / \mathrm{kg}$ enquanto o paciente estava internado, sendo posteriormente substituída pelo fluconazol na dose de 100 a $400 \mathrm{mg} /$ dia.

$\mathrm{Na}$ tentativa de se reduzirem as infecções por herpes foi administrado aciclovir do dia -1 ao D+26 na dose de $250 \mathrm{mg} / \mathrm{m}^{2}$ de $8 \mathrm{em} 8$ horas quando o doador e o paciente eram ambos sorologicamente citomegalovírus (CMV) negativos ou positivos, ou o doador era CMV negativo e o paciente era positivo. Utilizaram-se $500 \mathrm{mg} / \mathrm{m}^{2}$ de $8 \mathrm{em} 8$ horas de aciclovir quando o doador era CMV positivo e o paciente negativo.

Todos os derivados sangüíneos foram previamente irradiados com $2.500 \mathrm{cGy}$.

A falha de pega (rejeição) foi definida como:

1-Falha primária de pega (FPP): Ausência de recuperação hematológica em paciente sobrevivendo 21 dias ou mais após o transplante.

2- Pega transitória (PT): Recuperação completa ou par- cial da hematopoese originária do doador, seguida por recorrente pancitopenia, com uma medula óssea acentuadamente hipocelular e na ausência de DECH aguda moderada a severa.

Os pacientes sem evidência de pega até o $\mathrm{D}+25$ e alguns pacientes que apresentavam falha tardia receberam nova infusão de medula óssea precedida por outro condicionamento, constituído na maioria das vezes por ciclofosfamida associada à globulina antilinfocítica ou por ciclofosfamida e bussulfano. A opção por se indicar o segundo transplante ou o tratamento imunossupressor baseou-se na rapidez da rejeição e na gravidade da neutropenia. O tratamento imunossupressor somente era utilizado quando houvesse células hematopoéticas na medula óssea e os granulócitos não estivessem abaixo de $100 \mu \mathrm{L}$.

O tratamento imunossupressor consistiu de $12,5 \mathrm{mg} /$ $\mathrm{kg}$ de CSA por oito dias, $7 \mathrm{mg} / \mathrm{kg}$ do D+9 ao D+84 e, a seguir, $6,25 \mathrm{mg} / \mathrm{kg}$ durante um ano, associado a prednisona. Usavase esta última na dose de $2 \mathrm{mg} / \mathrm{kg}$ durante 15 dias, posteriormente administrava-se metade da dose por mais 15 dias, quando então era reduzida em $20 \%$ por semana.

\section{Análise Estatística}

A análise estatística foi feita com auxílio do programa Statgraphics plus versão 5 (Manugistics, Inc.). Foi adotado o limite de significância de $95 \%$ para as análises e testes estatísticos, ou seja, o p-valor inferior a 0,05 confirmando a significância das análises. As análises univariadas foram realizadas empregando-se a distribuição t-Student para a realização dos testes de hipóteses.

O modelamento estatístico multivariado foi realizado através da regressão linear múltipla entre a variável sobrevida (dependente) e as variáveis independentes: idade dos pacientes, número de transfusões prévias, desempenho pré-TMO, tipo de tratamento prévio da aplasia e o tipo de condicionamento, utilizando-se um limite de significância de $90 \%$.

As curvas de sobrevida foram analisadas usando-se o método de Kaplan e Meier.

\section{Resultados}

As características pré-transplante dos 178 pacientes são apresentadas na tabela 1 .

\section{Rejeição}

A rejeição ou falha de pega ocorreu em 39 dos 178 pacientes transplantados por anemia aplástica. Dos pacientes condicionados com ciclofosfamida, 24 (46\%) apresentaram rejeição, sendo três $(6 \%)$ com falha primária de pega (FPP) e 21 (40\%) com pega transitória (PT). Entre os pacientes condicionados com BU+CFA, 15 (12\%) apresentaram rejeição, sendo quatro (3\%) com FPP e 11 (9\%) com pega transitória (Tabela 2). 


\begin{tabular}{|c|c|c|c|}
\hline \multicolumn{4}{|c|}{$\begin{array}{c}\text { Tabela } 1 \\
\text { Características clínicas }\end{array}$} \\
\hline Características & $\begin{array}{l}\text { CFA } \\
\mathrm{N}=52\end{array}$ & $\begin{array}{c}C F A+B U \\
N=126\end{array}$ & $P$ - valor \\
\hline $\begin{array}{l}\text { Idade paciente (anos) } \\
\text { Variação/ Mediana }\end{array}$ & $\begin{array}{l}2-38 \\
M=16\end{array}$ & $\begin{array}{l}2-46 \\
M=19\end{array}$ & 0,017 \\
\hline Idade do doador (anos) & $\begin{array}{l}1-48 \\
M=16\end{array}$ & $\begin{array}{l}1-48 \\
M=19\end{array}$ & 0,140 \\
\hline $\begin{array}{c}\text { Sexo paciente } \\
\text { Masculino } \\
\text { Feminino }\end{array}$ & $\begin{array}{l}34(65 \%) \\
18(35 \%)\end{array}$ & $\begin{array}{l}80(63 \%) \\
46(37 \%)\end{array}$ & 0,812 \\
\hline $\begin{array}{c}\text { Raça } \\
\text { Branca } \\
\text { Não branca }\end{array}$ & $\begin{array}{l}46(89 \%) \\
6(11 \%)\end{array}$ & $\begin{array}{c}64(51 \%) \\
62(49)\end{array}$ & 0,000 \\
\hline $\begin{array}{l}\text { Duração da doença } \\
\text { (meses) }\end{array}$ & $\begin{array}{l}1-36 \\
M=2\end{array}$ & $\begin{array}{c}1-144 \\
M=4\end{array}$ & 0,009 \\
\hline $\begin{array}{l}\mathrm{N}^{\circ} \text { de transfusões } \\
\text { prévias (unidades) }\end{array}$ & $\begin{array}{l}1-30 \\
M=10\end{array}$ & $\begin{array}{c}15-675 \\
M=35\end{array}$ & 0,000 \\
\hline $\begin{array}{l}\text { Desempenho pré - TMO } \\
\text { (Escala de KarnofsKy) }\end{array}$ & $\begin{array}{l}70-100 \\
M=90\end{array}$ & $\begin{array}{c}30-100 \\
M=80\end{array}$ & 0,001 \\
\hline № granulócitos & $\begin{array}{l}22-1008 \\
M=278\end{array}$ & $\begin{array}{c}0-2072 \\
M=281\end{array}$ & 0,463 \\
\hline Infectados ao TMO & $6(11 \%)$ & $36(28 \%)$ & 0,005 \\
\hline $\begin{array}{l}\text { Etiologia } \\
\text { Idiopática } \\
\text { Drogas } \\
\text { Pós-hepatite }\end{array}$ & $\begin{array}{c}42(81 \%) \\
10(19 \%) \\
0\end{array}$ & $\begin{array}{c}115(91 \%) \\
9(7 \%) \\
2(2 \%)\end{array}$ & 0,118 \\
\hline $\begin{array}{c}\mathrm{N}^{\circ} \text { cél. Infundidas } \\
\left.\text { (x } 10^{*} 8 / \mathrm{kg} \text { receptor }\right) \\
<2,0 \\
\geq 2,0<2,5 \\
\geq 2,5<3,0 \\
\geq 3,0<4,0 \\
4,0 \\
\text { Mediana } \\
\text { Variação }\end{array}$ & $\begin{array}{c}1(2 \%) \\
12(23 \%) \\
12(23 \%) \\
15(29 \%) \\
12(23 \%) \\
2,97 \\
1,69-5,93\end{array}$ & $\begin{array}{c}14(11 \%) \\
26(21 \%) \\
26(21 \%) \\
41(32 \%) \\
19(15 \%) \\
2,87 \\
1,38-6,55\end{array}$ & 0,281 \\
\hline $\begin{aligned} \text { Sexo (doador } & \rightarrow \text { paciente) } \\
M & \rightarrow M \\
M & \rightarrow F \\
F & \rightarrow M \\
F & \rightarrow F\end{aligned}$ & $\begin{array}{l}34 \rightarrow 21 \\
34 \rightarrow 13 \\
18 \rightarrow 10 \\
18 \rightarrow 8\end{array}$ & $\begin{aligned} 80 & \rightarrow 55 \\
80 & \rightarrow 25 \\
46 & \rightarrow 31 \\
46 & \rightarrow 15\end{aligned}$ & 0,479 \\
\hline $\begin{array}{c}\text { Incompatibilidade ABO } \\
\text { Maior } \\
\text { Menor } \\
\text { Maior e Menor } \\
\text { Total }\end{array}$ & $\begin{array}{c}9(17 \%) \\
3(6 \%) \\
1(2 \%) \\
13(25 \%)\end{array}$ & $\begin{array}{c}15(12 \%) \\
25(20 \%) \\
4(3 \%) \\
44(35 \%)\end{array}$ & 0,783 \\
\hline $\begin{array}{c}\text { Tratamento prévio } \\
\text { Prednisona } \\
\text { Prednisona + ciclosporina } \\
\text { Prednisona + outros } \\
\text { Miscelânea } \\
\text { Nenhum }\end{array}$ & $\begin{array}{c}5(9 \%) \\
3(6 \%) \\
3(6 \%) \\
1(2 \%) \\
40(77 \%)\end{array}$ & $\begin{array}{c}16(13 \%) \\
24(19 \%) \\
12(9 \%) \\
6(5 \%) \\
68(54 \%)\end{array}$ & 0,067 \\
\hline
\end{tabular}

Em relação aos 52 pacientes condicionados com CFA comparamos as suas características clínicas em função da média dos possíveis fatores relacionados à rejeição dos 24 pacientes que apresentaram rejeição e dos 28 que não apresentaram. Esta diferença foi estatisticamente significativa em relação à idade do paciente $(\mathrm{p}=0,007)$, idade dos doadores $(p=0,022)$, etiologia da anemia aplástica $(p=0,023)$ e tratamento prévio da aplasia $(\mathrm{p}=0,013)$. Ainda em relação aos pacientes condicionados com CFA temos que os pacientes que rejeitaram eram mais jovens $(M=10)$ que os que não rejeitaram ( $M=17$ anos), bem como seus doadores $(M=14$ anos para os doadores de pacientes com rejeição $X \mathrm{M}=20$ anos para os doadores dos pacientes sem rejeição). Predominam os pacientes com AA idiopática e os tratados com prednisona somente ou associada a ciclosporina dentre os pacientes sem rejeição.

Ao compararmos as médias dos possíveis fatores relacionados à rejeição dos 126 pacientes condicionados com a combinação de ciclofosfamida e bussulfano, separando-se em um grupo de 15 pacientes que apresentaram rejeição e o grupo dos 111 que não apresentaram, obtivemos uma diferença estatisticamente significativa apenas em relação à média da idade do paciente ao transplante de medula $(\mathrm{p}=0,0315)$. Os pacientes com rejeição eram mais jovens $(\mathrm{M}=11 \mathrm{X} \mathrm{M}=20$ anos). Em relação aos outros fatores temos: idade do doador $(\mathrm{p}=0,7841)$, intervalo entre o diagnóstico e o transplante $(\mathrm{p}=$ $0,4107)$, número de transfusões prévias ( $\mathrm{p}=0,4909)$, desempenho pré-TMO $(\mathrm{p}=0,5732)$, número de células mononucleares infundidas $(\mathrm{p}=0,3746)$, número de neutrófilos ao transplante $(p=0,1053)$, raça $(p=0,8356)$, número de pacientes com infecção ao condicionamento $(p=0,666)$, tratamento prévio da aplasia $(\mathrm{p}=0,9190)$, relação entre o sexo do doador e do receptor $(\mathrm{p}=0,2890)$ e incompatibilidade $\mathrm{ABO}(\mathrm{p}=0,0586)$. Não foi possível comparar os dados referentes à etiologia da AA nestes dois grupos, pois foram considerados iguais do ponto de vista estatístico.

\section{Sobrevida}

Quando se observa a estimativa de sobrevida para os pacientes que rejeitaram ( 24 dos condicionados com CFA e 15 dos condicionados com BU+CFA) observamos uma diferença expressiva de resultados. Os pacientes inicialmente condicionados com CFA e que rejeitaram tiveram uma sobrevida de aproximadamente $80 \%$, enquanto os pacientes condicionados com BU+CFA, de aproximadamente $35 \%$. Há uma diferença estatisticamente significante entre as curvas $(\mathrm{p}=0,0017)$ (Figura 1$)$.

Inferimos que os pacientes politransfundidos condicionados com BU+CFA, embora apresentem um menor índice de rejeição, cursam com uma maior dificuldade de salvamento pós-rejeição.

A análise da sobrevida considerando-se os pacientes com pega transitória $(n=32)$ e dos pacientes com falha pri- 


\begin{tabular}{cccc}
\multicolumn{4}{c}{ Tabela 2} \\
Índice de rejeição conforme o condicionamento usado \\
\hline Condicionamento & $\begin{array}{c}\text { Total de pacientes } \\
(\%)\end{array}$ & $\begin{array}{c}\text { Falha primária } \\
\text { de pega (FPP) }\end{array}$ & $\begin{array}{c}\text { Pega transitória } \\
(\mathrm{PT})\end{array}$ \\
\hline $\begin{array}{c}\text { Ciclofosfamida } \\
\mathrm{N}=52\end{array}$ & $24(46 \%)$ & $3(6 \%)$ & $21(40 \%)$ \\
$\begin{array}{c}\text { Bussulfano }+ \\
\text { ciclofosfamida } \\
\mathrm{N}=126\end{array}$ & $15(12 \%)$ & $4(3 \%)$ & $11(9 \%)$
\end{tabular}

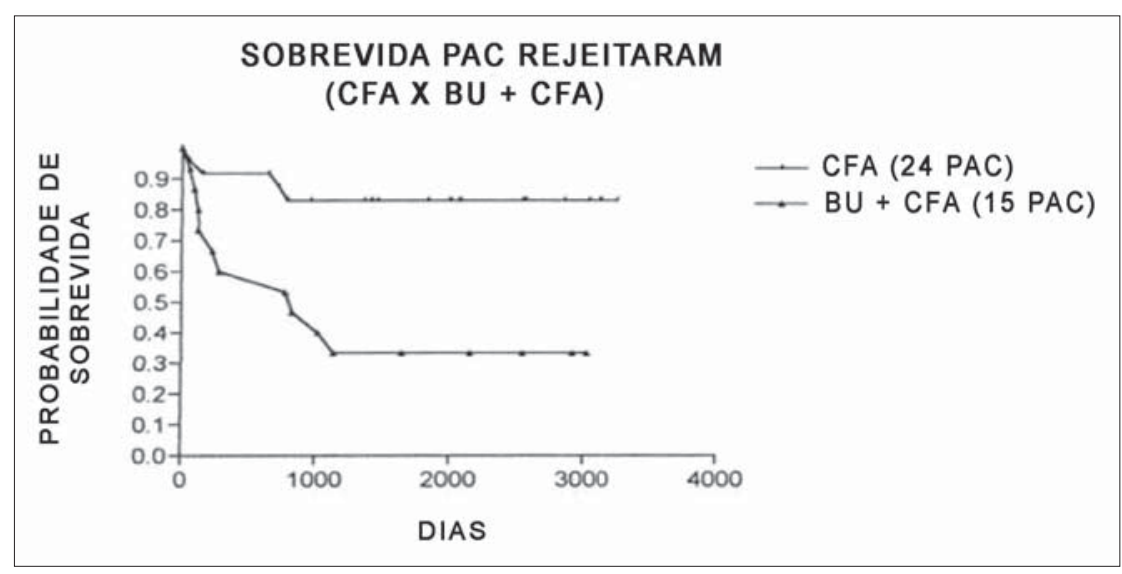

Figura 1. Estimativa de sobrevida dos pacientes que rejeitaram conforme o regime de condicionamento usado. Método de Kaplan-Meier

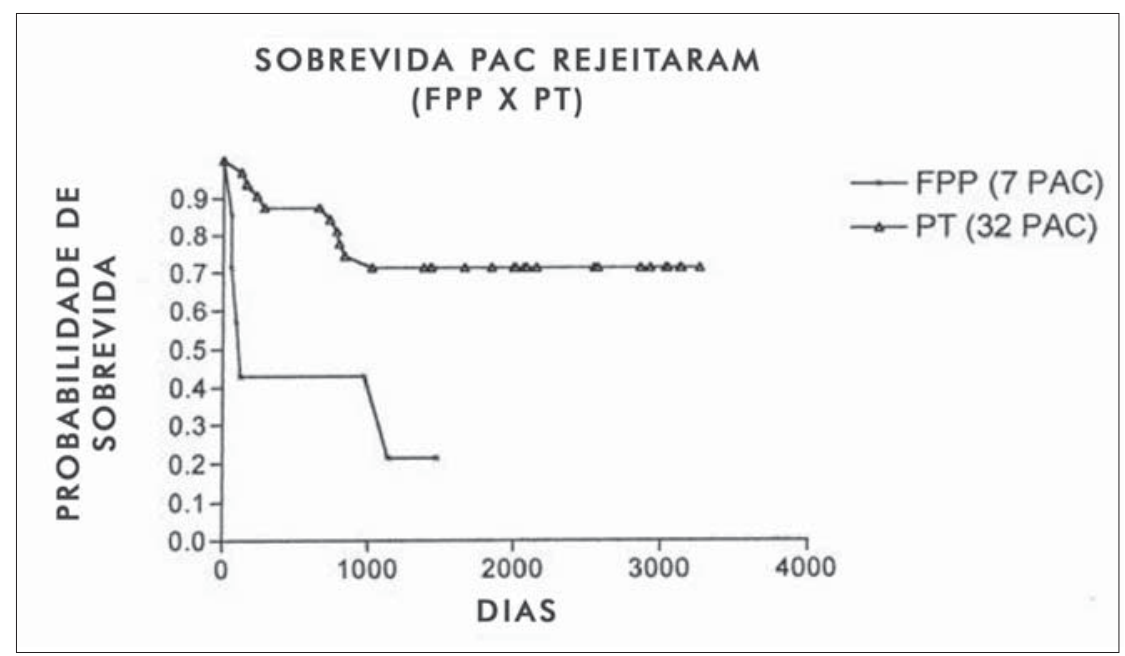

Figura 2. Estimativa de sobrevida dos pacientes que apresentaram falha primária de pega (FPP) e pega transitória (PT). Método Kaplan-Meier

mária de pega $(\mathrm{n}=7)$ mostra que a ocorrência desta última compromete o sucesso do TMO (Figura 2).

A superioridade nos resultados da sobrevida dos pacientes com pega transitória é expressiva quando se compara com os resultados dos pacientes com FPP (70\% x 20\% após mil dias). Há significância estatística entre as duas curvas $(\mathrm{p}=0,002)$.

\section{Discussão}

O TMO alogênico é hoje aceito como uma modalidade terapêutica para o tratamento de pacientes com AAS que possuem um doador aparentado compatível. Apesar da taxa de sobrevida destes pacientes estar entre $60 \%$ e $80 \%$, temos alguns problemas a serem resolvidos. ${ }^{12}$ Diferente do que ocorre no tratamento das leucemias agudas e crônicas, a rejeição permanece como um problema significativo para os pacientes com AAS após o transplante. Vários fatores contribuem para a possibilidade de rejeição, incluindo a transfusão prévia de hemácias e plaquetas, ${ }^{13}$ a infusão de pequena quantidade de células do doador, ${ }^{14}$ o grau de disparidade dos antígenos HLA e a natureza auto-imune da doença de base. ${ }^{15}$

A administração do creme leucocitário (buffy coat cells) do doador ${ }^{16}$ e a administração de esteróides também têm mostrado alterar o risco de rejeição. ${ }^{17}$ Estratégias empregadas na profilaxia da DECH, como a depleção de células T, também contribuem para o risco de rejeição. ${ }^{17}$ Finalmente, a adição de irradiação ao regime de condicionamento aumenta as chances de pega. ${ }^{17} \mathrm{O}$ prognóstico após a rejeição é ruim, apesar de, em um pequeno número de casos, detectar-se recuperação autóloga. ${ }^{18}$

O Serviço de TMO do HC da UFPR acumula a experiência de 178 casos de AAS transplantados desde 1993, usando como condicionamento a CFA ou a combinação desta ao bussulfano. Todos os pacientes receberam medula óssea repleta de células $\mathrm{T}$ e, como imunoprofilaxia para a $\mathrm{DECH}, \mathrm{o}$ methotrexate associado a ciclosporina. Esta experiência reveste-se da maior importância, pois apresenta uma casuística expressiva para uma doença rara e avalia o índice de rejeição nos pacientes condicionados com BU+CFA, o que é uma casuística ímpar na literatura. Com a melhora da sobrevida após o transplante ao longo do tempo ${ }^{19}$ podemos avaliar qual o impacto atual da rejeição nesses resultados.

As características clínicas da nossa casuística assemelham-se aos outros estudos de porte semelhante. A etiologia da AA, a idade mediana dos pacientes, as características dos doadores, a incompatibilidade $\mathrm{ABO}$, o número de transfusões prévias, o tratamento da aplasia pré-transplante são 
similares aos estudos publicados na literatura médica. O intervalo entre o diagnóstico da AAS e a realização do TMO foi de três meses, sendo que apenas 18 pacientes (10\%) foram transplantados no primeiro mês de diagnóstico. No estudo do IBMTR, 20\% dos casos estavam diagnosticados a menos de um mês quando transplantados, e a análise estatística destes dados demonstrou que o risco de insucesso do TMO (morte por qualquer causa) é significante para aqueles com mais de um mês de diagnóstico.$^{20}$ Em nosso país, o atraso na realização do TMO deve-se à baixa capacidade de execução do procedimento e aos obstáculos gerados pelos enormes problemas socioeconômicos.

As transfusões sangüíneas administradas antes do transplante são fatores desfavoráveis para a sobrevida. Os resultados do TMO nos pacientes não transfundidos são claramente superiores, pois o índice de rejeição é menor. ${ }^{21,22}$ Esta superioridade dos resultados também é nítida no grupo recebendo até vinte transfusões na análise feita pelo IBMTR. ${ }^{20}$ No serviço de TMO da UFPR ficou estabelecido o limite de corte de 15 transfusões, baseado nos dados da tese defendida pelo Dr. Ricardo Pasquini para o concurso de professor titular, que mostrava uma sobrevida de $74 \%$ naqueles pacientes que receberam um menor número de unidades e de $46 \%$ para os que receberam mais de 15 unidades de hemoderivados. ${ }^{23}$ Nos países mais desenvolvidos, os pacientes portadores de AA são referidos aos centros de transplante mais precocemente e conseqüentemente com menor exposição às transfusões. ${ }^{24} \mathrm{~A}$ alternativa de se exporem as frações do sangue a serem transfundidas a radiação ionizante foi proposta para reduzir a influência das transfusões na evolução do TMO. ${ }^{25}$

O grupo dos 52 pacientes condicionados com CFA e os 126 condicionados com BU+CFA diferiram em relação à idade dos pacientes, à raça, à duração da doença, ao número de transfusões prévias, ao desempenho pré-TMO, ao número de pacientes infectados previamente ao condicionamento e quanto ao tipo de tratamento prévio ao transplante. Alguns fatores podem estar interligados, como a maior duração da doença ter como conseqüência um maior número de transfusões e um maior risco de infecções, com uma conseqüente piora no desempenho.

No grupo condicionado com BU+CFA há maior número de pacientes submetidos ao tratamento prévio com ciclosporina e prednisona; esta tentativa de tratamento imunossupressor pode atrasar o transplante, tendo em vista que a resposta terapêutica é avaliada em média com quatro semanas de tratamento. ${ }^{26}$

Não houve diferença estatisticamente significante em relação ao sexo dos pacientes, ao número de granulócitos, à etiologia da hepatite, ao número de células mononucleares infundidas, à relação entre o sexo do paciente e do doador e à incompatibilidade $\mathrm{ABO}$.

A incidência de rejeição entre os pacientes condicionados com CFA foi alta (46\%), tendo $6 \%$ dos pacientes apre- sentado FPP e 40\% pega transitória. No estudo realizado pelo IBMTR, ${ }^{27}$ o grupo comparável com a nossa estatística, isto é, aquele condicionado com CFA isolada, apresentou 18\% de rejeição. Dr. Ricardo Pasquini, quando avaliou 108 pacientes transplantados com AAS no HC da UFPR, tendo 100 pacientes desse grupo recebido $200 \mathrm{mg} / \mathrm{kg}$ de ciclofosfamida e os oito restantes a associação desta às diferentes doses de radioterapia, verificou a incidência de rejeição em 26 de 104 pacientes, isto é $25 \%{ }^{28}$

Entre os fatores que podem reduzir o risco de rejeição incluem-se a transfusão de hemácias depletadas de leucócitos e o uso de plaquetas de doador único. ${ }^{29}$ Os estudos mais recentes em modelos animais mostram que o risco de sensibilização a antígenos menores (mas não aos maiores) de histocompatibilidade podem ser praticamente eliminados quando os produtos sangüíneos são expostos a irradiação gama (2000 cCy). ${ }^{30}$ Estes dados experimentais sugerem que todo produto sangüíneo deve ser irradiado antes de ser transfundido, antes e após o transplante.

A prática de irradiar os hemoderivados, bem como a leucodepleção, não faz parte da realidade da maioria dos hemobancos brasileiros, seja por falta de acesso a serviços bem estruturados, seja por problemas econômicos. O Hospital de Clínicas da UFPR, em Curitiba, iniciou a irradiação de hemoderivados em 1989. Utilizamos o filtro desde 1997 para os pacientes politransfundidos ou com indicação de TMO, porém este uso não é sistemático. Não há uma regulamentação por parte das autoridades e não há pagamento pelo Sistema Único de Saúde (SUS), fato que explicaria em parte o alto índice de rejeição dos pacientes condicionados somente com ciclofosfamida. Em contrapartida, a intensificação do regime de condicionamento funciona como um fator a mais na tentativa de minimizar a rejeição. ${ }^{31}$ Isto pode explicar termos apenas $12 \%$ de rejeição entre os 126 pacientes condicionados com a associação de CFA+BU.

A quantidade de células mononucleares infundidas não foi associada à rejeição, entretanto $91 \%$ dos pacientes receberam mais do que $2,0 \times 10^{8}$ células $/ \mathrm{kg}$. Se doses menores do que essa podem estar associadas à falha de pega não se sabe.

O tratamento da aplasia com corticosteróide antes de referir o paciente ao transplante foi associado à baixa incidência de falha do enxerto. A explicação para esse fato não é clara e também foi observada em outro trabalho do IBMTR. ${ }^{32}$ O corticóide inibe a imunidade celular e humoral nos pacientes com AA e a pega pode ser facilitada por este mecanismo. ${ }^{33}$

Os pacientes com falha primária de pega estão associados a um pior prognóstico. No Hospital de Clínicas, sete pacientes não tiveram evidência de pega e, dentre estes, somente um sobreviveu ao segundo TMO. Esse subgrupo é pequeno e não apresentou uma curva de distribuição normal. Não estão definidos na literatura os fatores relacionados à FPP e nós também não conseguimos evidenciar uma 
diferença estatística entre os fatores relacionados a este evento. A possibilidade de um defeito no microambiente da medula óssea tem sido considerada nesses casos em que a pega do enxerto não se faz, mesmo com mais de um condicionamento.

Dentre os 39 pacientes que apresentaram rejeição, vinte foram retransplantados (dez pertencem ao grupo inicialmente condicionado com CFA e dez ao grupo do BU+CFA) e dez pacientes foram submetidos a tratamento imunossupressor. A evolução desses pacientes, isto é, a proporção entre pacientes vivos e mortos foi praticamente a mesma independente do tipo de tratamento pós-rejeição.

A sobrevida após o TMO para os pacientes com anemia aplástica tem melhorado nos últimos 15 anos, ${ }^{34}$ possivelmente devido ao uso de novos antibióticos e antifúngicos, ao uso da ciclosporina na profilaxia da $\mathrm{DECH}^{34}$ e a outros fatores difíceis de mensurar. Pelo apresentado, não podemos associar a melhor sobrevida a um menor índice de rejeição nos últimos anos, o que nos faz sugerir o uso sistemático do filtro, da leucodepleção e da irradiação dos hemoderivados para os pacientes candidatos ao TMO.

\section{Conclusões}

O regime de condicionamento usando ciclofosfamida (200 mg/kg) é eficaz para o tratamento dos pacientes com anemia aplástica. Apesar do alto índice de rejeição apresentado pelos pacientes (46\%), a sobrevida daqueles que rejeitam também é alta (aproximadamente $80 \%$ ), pois conseguem ser resgatados por um novo transplante ou pelo tratamento imunossupressor com ciclosporina.

$\mathrm{O}$ regime de condicionamento com a associação de ciclofosfamida e bussulfano é eficaz para os pacientes politransfundidos transplantados por anemia aplástica. Apresenta um índice de rejeição de $12 \%$ e uma sobrevida global de aproximadamente $75 \%$. Os pacientes que rejeitam apresentam uma sobrevida de aproximadamente $35 \%$.

A falha primária de pega está relacionada a um mal prognóstico, não havendo diferença estatística entre as médias das variáveis estudadas relacionadas à falha primária de pega e a não rejeição.

O índice de rejeição para os pacientes transplantados por anemia aplástica continua alto no nosso país, ao contrário do que se tem observado nos países desenvolvidos. Isso implica a necessidade de criação de novos centros de transplante de medula óssea e na agilização do procedimento, diminuindo o número de transfusões pré-TMO, bem como na padronização do uso de filtros e irradiação dos hemoderivados para os pacientes candidatos a transplante.

\section{Abstract}

Bone marrow transplantation is an effective therapy for severe aplastic anemia and is generally considered the preferable treatment for young patients who have an HLA (Human Leukocyte Antigen) identical sibling donor. Recent studies report $55 \%$ to $80 \%$ extended survival. Graft failure owing to rejection or others causes remains an important life-threatening complication following allogeneic bone marrow transplantation for aplastic anemia. It occurs in 55\% to $60 \%$ of patients receiving HLA identical transplants, using different immunosuppressive therapies before and after transplant. The BMT Unit of Federal University of Paraná had 178 cases of SAA transplants from 1993 to 2001 using either cyclophosphamide (CY) alone or associated with busulfan $(C Y+B U)$ for conditioning regimen. Graft failure occurred in 39 of the cases. Among patients conditioned with CY, 24 (46\%) presented graft failure, of whom 3 (6\%) suffered primary graft failures and 21 (40\%) transient engraftment. Among patients conditioned with $B U+C Y, 15(12 \%)$ suffered graft failure, 4 (3\%) primary graft failures and 11 (9\%) transient engraftment. The patients conditioned with $C Y$ and experienced rejection had a survival of around $80 \%$, because they underwent another transplantation or due to immunosuppressive treatment with cyclosporin A. The patients conditioned with $B U+C Y$ who suffered rejections had a survival of approximately $35 \%$. Rev. bras. hematol. hemoter. $2005 ; 27(1): 5-11$.

Key words: Bone marrow transplantation; severe aplastic anemia; rejection; primary graft failure; cyclophosphamide; busulfan.

\section{Referências Bibliográficas}

1. Bortin MM, Gale RP, Rimmm AA. Allogeneic bone marrow transplantation for 144 patients with severe aplastic anemia. JAMA 1981;245:1132.

2. Camitta BM, Storb R, Thomas ED. Aplastic anemia: Pathogenesis, diagnosis, treatment and prognosis. N Engl J Med 1982;306:645.

3. Champlin RE. Treatment of aplastic anemia, in Gale RP, Champlin RE (eds). Progress in bone marrow transplantation. New York, Liss, 1987, p37.

4. Feig SA, Champlin RE, Gale RP et al. An improved survival following bone marrow transplantation for aplastic anemia. $\mathrm{Br} \mathrm{J}$ Haematol 1983;54:509.

5. McGlave PB, Haake R, Miller W et al. Therapy of severe aplastic anemia in young adults and children with allogeneic bone marrow transplantation. Blood 1987;70:1325.

6. Elfenbein GL, Mellitus ED, Santos GW. Engraftment and survival after allogeneic bone marrow transplantation for severe aplastic anemia. Transplant Proc 1983;15:1412.

7. Storb R, Thomas ED, Buckner CD et al. Marrow transplantation in thirty untranfused patients with severe aplastic anemia. Ann Intern Med 1980;92:30.

8. Baciagalupo A, Gordon-Smith EC, Van Lint MT et al. Bone marrow transplantation versus immunosuppression in the management of severe aplastic anemia. Bone Marrow Transplant 1987 (suppl 1); 2:99.

9. Champlin RE, Feig SA, Ho WG. Graft failure following bone marrow transplantation: Its biology and treatment. Exp Hematol 1984;12:728.

10. Storb R, Prentice RL, Thomas ED. Factors associated with graft rejection after HLA identical marrow transplantation for aplastic anemia. Br J Haematol 1983;55:573.

11. Camita BM, Thomas ED, Nathan DG, et al. Severe aplastic anemia: A prospective study of the effect of early marrow transplantation on acute mortality. Blood 1976;48:63-70.

12. Vogelsang B. How I treat chronic graft-versus-host disease. Blood 2001;97:1196-1201. 
13. Neiderwieser D, Pepe M, Storb R et al. Improvement in rejection, engraftment rate and survival without increase in graft-versushost disease by high marrow cell dose in patients transplanted for aplastic anaemia. Br J Haematol 1988;69:23-28.

14. Storb R, Champlin RE. Bone marrow transplantation for severe aplastic anemia. Bone Marrow Transplant 1991; 8:69-72.

15. Champlin RE, Feig SA, Sparkes RS et al. Bone marrow transplantation from identical twins in the treatment of aplastic anaemia: implications for the pathogenesis of the disease. $\mathrm{Br} \mathrm{J}$ Haematol 1984;56:455-463.

16. Storb R, Doney KC, Thomas ED et al. Marrow transplantation with and without donor Buffy coat cells for transfused aplastic anemia patients. Blood 1982;59:236-246.

17. Champlin RE, Horowitz MM et al. Graft failure following bone marrow transplantation for severe aplastic anemia: risk factors and treatment results. Blood 1989; 73:606-613.

18. Storb R, Weiden PL, Sullivan KM et al. Second marrow transplants in patients with aplastic anemia rejecting the first graft: use of a conditioning regimen including cyclophosphamide and antithymocyte globulin. Blood 1987; 70:116-121.

19. Passweg JR, Socié G, Hinterberger W et al. Bone marrow transplantation for severe aplastic anemia: Has outcome improved? Blood 1997;90:858-864.

20. Gluckman E, Horowitz MM, Champlin RE et al. Bone marrow transplantation for severe aplastic anemia: influence of conditioning and graft-versus-host disease prophylaxis regimens on outcome. Blood 1992;79:269-275.

21. Storb R, Thomas ED, Buckner CD et al. Marrow transplantation in thirty untransfused patients with severe aplastic anemia. Ann Intern Med 1980;92:30-36.

22. Anasetti C, Doney KC, Sorb R et al. Long-term outcomes in fifty untransfused patients. Ann Intern Med 1986;104;361-366.

23. Mcglave PB, Haake R, Kim T et al. Therapy of severe aplastic anemia in young adults and children with allogeneic bone marrow transplantation. Blood 1987;70:1325-1330.

24. Nissen C, Tichelli A, Gratwhol A et al. Failure of recombinant therapy in aplastic anemia patients with very severe neutropenia. Blood 1988;72:2.045-2.047.

25. Deeg HJ, Aprile J, Graham TC et al. Ultraviolet irradiation of blood prevents transfusion induced sensitization and marrow graft rejection in dogs. Blood 1986;67:537-539.

26. Frickhofen N, Rosenfeld SJ. Immunosuppressive treatment of aplastic anemia with antithymocyte globulin and cyclosporine. Seminars in Hematology 2000;37(1):56-68.

27. Champlin RE, Horowitz MM, Van Bekkum et al. Graft failure following bone marrow transplantation for severe aplastic anemia: risk factors and treatment results. Blood 1988;73: 606613 .

28. Pasquini R. Transplante de medula óssea na anemia aplástica severa. Curitiba,1991. Tese (Professor Titular do Departamento de Clínica Médica) - Setor de Ciências da Saúde, Universidade Federal do Paraná

29. Storb R, Deeg HJ. Failure of allogeneic canine marrow grafts after total body irradiation: Allogeneic "resistance" vs. transfusion induced sensitization. Transplantation 1986;42: 571-580.

30. Bean MA, Storb R, Grahan T et al. Prevention of transfusioninduced sensitization to minor histocompatibility antigens on DLAidentical canine marrow grafts by gamma irradiation of marrow donor blood. Transplantation 1991;52:956-960.

31. Deeg HJ. Graft failure. In: Burt RK, Deeg HJ, Lothian ST, et al. On call in... bone marrow transplantation. Austin: R.G. Landes Company, 1996. p.312.

32. Champlin RE, Horowitz MM, van Bekkum et al. Graft failure following bone marrow transplantation for severe aplastic anemia: risk factors and treatment results. Blood 1989;73 (2):606-613.
33. Elfenbein GJ, Clayton HK, Tutschka PJ et al. The immune system in 40 aplastic anemia patients receiving conventional therapy. Blood 1979;53:652.

34. Passweg JR, Socié G, Hinterberger W et al. Bone marrow transplantation for severe aplastic anemia: has outcome improved? Blood 1997;90(2):858-864.

Avaliação: Editor e dois revisores externos. Conflito de interesse: não declarado

Recebido: $18 / 02 / 2005$

Aceito após modificações: 15/03/2005 